\title{
METHODS \& DESIGNS Ratings of the auditory and visual similarity of consonants: Implications for research
}

\author{
SUSAN KARP MANNING \\ Hunter College of the City University of New York, New York, New York 1002I
}

\begin{abstract}
A set of rating data was collected which compared the visual similarity of consonants to the auditory similarity of the same letters. Analysis of the rating patterns raises important questions about the use of similarity data in drawing conclusions about mode of memory coding in a variety of situations.
\end{abstract}

Although a large amount of research has been based on assumptions about the auditory and visual similarity of letters (e.g., Connor \& Hoyer, 1976; Vingilis, Blake, \& Theodor, 1977), it appears that no data are available which compare the visual similarity of alphabetic graphemes to the auditory similarity of the same letters. Thus, there is no way of knowing whether alphabetic characters are more auditorily or more visually confusing.

Some relevant information is available in Conrad (1964), in which data are presented on the number and type of confusions which occur when letters are presented auditorily under degraded listening conditions. Additionally, Gibson (1969) has analyzed graphemes according to a set of 12 distinctive features, which yield a unique configuration for each letter. The results suggest that letters with a high proportion of common features tend to be confused more of ten than those which do not. Since confusion suggests similarity, it is probable that both the Conrad and the Gibson analyses are tapping letter similarity.

Although these measures are useful for comparing and manipulating either graphemic or phonemic similarity, they cannot be used to compare the two because phonemes may be more similar to each other than are graphemes. Consider three letter sets, one of high phonemic similarity, one of high graphemic similarity, and one a control condition low on both variables. Performance decrements are frequently, although not always (e.g., Vingilis et al., 1977), found for the high phonemic similarity set but not for the high graphemic similarity set, when compared to the control group in a wide variety of tasks (e.g., Connor \& Hoyer, 1976).

Such results have led theorists to conclude that information is auditorily rather than visually encoded in the situations in question. While this may be a valid conclusion, it is also possible that identical data result if both types of coding exist and graphemes

I wish to thank Linda Sapin for her work in collecting and evaluating the data for this project. This research was supported by Grant BNS-76-18683 from the National Science Foundation to the author. are less similar than are phonemes.

In order to provide information about graphemic and phonemic similarity, a set of specially designed ratings was collected.

\section{METHOD}

\section{Subjects}

The subjects were 28 male and female Hunter College students who participated in two groups during class sessions of two separate sections of experimental psychology.

\section{Stimulus Materials and Procedure}

During the experimental session, each student was given a booklet containing three parts. In Part 1, half of the subjects were asked to judge pairs of English consonants for sound similarity and half were asked to judge the same pairs for visual similarity. Subjects receiving the auditory task were told to make comparisons on the basis of the sound of the name of the letter, not on the basis of its phonetic usage. Subjects judging letters according to visual similarity were asked to use the letters as printed in the booklet as their standard.

Both groups rated on a 5-point scale printed in the booklet. Ratings ranged from +2 (very similar) to -2 (very dissimilar), with $O$ designated as neutral (i.e., neither similar nor dissimilar).

On the rating sheet, all possible paired combinations of consonants (except $Z$ ) were arranged in a random order. Thus, subjects were asked to make 190 ratings. All letter pairs were printed with a Pickett $16 \mathrm{~V}$ letter guide and a black pilot razor-point pen. Uppercase letters were $.48 \mathrm{~cm}$ in height and of a "modern light vertical" style. The original letters produced with the lettering guide were then Xeroxed for distribution to the subjects. Letter pairs were arranged in six columns on a single sheet of standard $21.6 \times 27.9 \mathrm{~cm}$ paper. Subjects wrote responses on a dash next to each letter pair.

Part 2 of the questionnaire consisted of the same task for all subjects. Stimuli, prepared using the same letter guide as in Part 1, consisted of 30 sets of consonant letter triplets. Ten triplets each were chosen randomly from groups of consonants thought to be auditorily similar, visually similar, and dissimilar on both dimensions. These sets consisted of B, C, D, G, P, T, V; K, M, N, R, W, X, Y; and F, H, J, L, Q $S, X$, respectively. Subjects were asked to assign each letter triplet to one of three categories: letter names sound alike, letters look alike, or letters are dissimilar.

On Part 3, all subjects were asked to divide the 21 English consonants into three sets consisting of letters which sounded alike, which looked alike, and which were dissimilar, respectively. Each letter was to be used only once; thus, each set was to contain seven letters. 

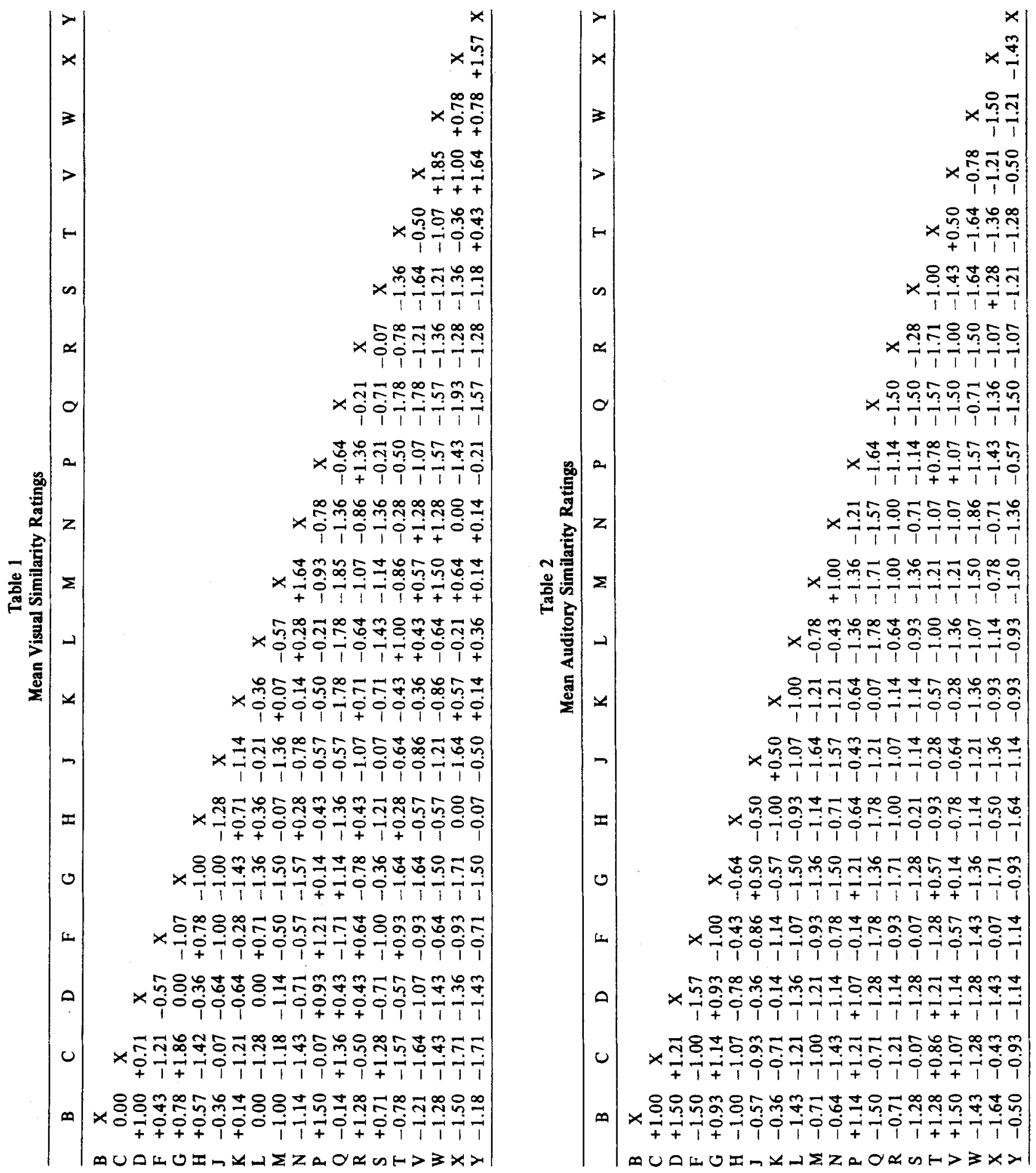


\section{RESULTS}

All statistical tests are reported at the .05 significance level. Matrices containing the mean visual and auditory ratings for each of the letter pairs are presented as Tables 1 and 2, respectively.

As a validation procedure, a Pearson product-moment correlation was computed between rated visual similarity and number of common features, using the Gibson (1969) analysis. The significant correlation, based on 30 randomly chosen letter pairs $\operatorname{~r}(28)=.65]$, showed that the ratings and the distinctive feature analysis shared a substantial amount of common variance.

A second correlation was computed between the auditory ratings and the Conrad (1964) confusion data using a different random sample of 30 letter pairs. The computed correlation was also reliable $[\mathrm{r}(28)=$ $.71]$, suggesting that the auditory confusion data probably tap much of the same variance as the rating data.

Thus, in common with Conrad (1964) and Gibson (1969), the current paper provides two sets of data, one for comparing the phonemic and one for comparing the graphemic similarity of letters. Unlike the earlier researchers (because the same scale was used for the two types of ratings), the data collected here also allow comparisons between the degree of phonemic and graphemic similarity. For example, the pair $\mathrm{BC}$ has a higher rating on the phonemic than on the graphemic scale.

Using this property of comparability, the rating patterns may be examined with respect to the two types of similarity. Over 50 pairs of letters were rated as more than 0.0 on the graphemic scale, while only 25 of the phonemic ratings were rated in that range. Of the 25 positive auditory similarity ratings, 21 were in the rhyming set $\mathrm{B}, \mathrm{C}, \mathrm{D}, \mathrm{G}, \mathrm{P}, \mathrm{T}, \mathrm{V}$, in which all pairs were positive. The remaining four pairs were JG, $\mathrm{JK}, \mathrm{XS}$, and $\mathrm{MN}$. No comparable visual set existed, although the set $K, M, N, R, W, X, Y$ had the highest interratings of a set of seven letters. Further, there were large numbers of positive visual ratings scattered through the matrix.

In order to demonstrate various aspects of the rating pattern, three letter sets were constructed. These consisted of an auditory (A) set, the seven consonants with the highest phonemic similarity interratings (B, C, D, G, P, T, V); a visual (V) set, the seven consonants with the highest graphemic similarity interratings (K, $\mathrm{M}, \mathrm{N}, \mathrm{R}, \mathrm{W}, \mathrm{X}, \mathrm{Y}$ ); and a neutral (N) set, the remaining six consonants used in our task $(\mathrm{F}, \mathrm{H}, \mathrm{J}, \mathrm{L}, \mathrm{Q}, \mathrm{S})$.

The mean interblock rating was taken for each of the three blocks for each subject. For example, for the phonemic similarity block, all combinations of ratings among $B, C, D, G, P, T$, and $V$ were summed and the mean computed. Table 3 contains the mean ratings over subjects for the graphemic and phonemic rating tasks.
The data were used in a 2 (task) by 3 (letter sets) analysis of variance. The rating group main effect was not significant $(F<1)$. There were significant effects for letter set $[F(2,52)=70.13]$ and the interaction of Task by Letter Set $[F(2,52)=76.93]$. The letter set main effect was largely due to the extremely high phonemic ratings in combination with the relatively high graphemic ratings for the $A$ set. The interaction was to a great degree due to the reversal of rankings of the $A$ and $V$ sets for the phonemic and graphemic raters. Thus, a readily identifiable set of letters with high phonemic similarity and a much less highly similar visual set was apparent. Additionally, the $\mathbf{A}$ set had a fair degree of graphemic similarity as compared, for example, to the $\mathrm{N}$ set.

The data from the second rating task were consistent with those already reported. Subjects were asked to assign 30 letter triplets to one of three categories: names of letters sound alike, letters look alike, or letters are dissimilar. Of 10 opportunities, subjects assigned a mean of 9.75 from the $A$ set to the first category, 5.78 from the $V$ set to the second category, and 7.85 from the neutral set to the third category. An analysis of variance showed a highly significant difference in hit rates for the three letter groups $[F(2,27)=25.27]$. Of importance in this comparison was the much greater number of assignments of the A letters to the sound-alike category than of the V letters to the look-alike category.

Part 3 provides further corroboration of the greater ease in identifying an auditory, as compared with a visual, similarity component in letter sets. When subjects were asked to construct three nonoverlapping sets of seven letters (one auditorily similar, one visually similar, and one dissimilar), the mean hit rates for the $A, V$, and $N$ sets were $6.46,5.32$, and 4.71 , respectively. A matched $t$ test showed the A set to be significantly better identified than the $V$ set $[t(27)=5.55]$. These data indicate that subjects also were able to find letters that were visually similar with a large degree of agreement.

\section{DISCUSSION}

This paper provides data useful for comparing letter similarity. The data have variance in common with

Table 3

Mean Ratings of the Auditory, Visual, and Neutral Sets on the Phonemic and Graphemic Similarity Rating Tasks

\begin{tabular}{lccc}
\hline & \multicolumn{3}{c}{ Set } \\
\cline { 2 - 4 } & Auditory & Visual & Neutral \\
\hline Phonemic Task & +1.02 & -1.12 & -.93 \\
Graphemic Task & -.26 & +.15 & -.68 \\
\hline
\end{tabular}

Note-The scale ranges from +2 (very similar) to -2 (very dissimilar), with 0 being designated neither similar nor dissimilar. 
the auditory confusability measures of Conrad (1964) and the visual distinctive feature analysis of Gibson (1969). However, unlike previous work, the scales used here allow comparisons of the degree of graphemic and phonemic similarity to be made.

The comparability of the scales and the analysis presented here have important implications. First, it appears that it is possible to construct an auditory set which has much higher interletter similarity ratings than any visual set that can be constructed. This set will be readily constructed by subjects themselves if they are asked to do so (Part 3). Additionally, subjects will identify letter triplets from this set with a very high probability (Part 2 ).

Apparently, then, the names of these alphabetic consonants sound more confusing than any set of graphemes looks. One could readily imagine an alphabet which contained a highly similar set of seven graphemes, e.g., B, G, B, P, P, G , b. The English alphabet, however, does not contain such a grouping.

This differential confusability suggests the need for caution in interpreting the meaning of performance deficits in auditorily and visually similar sets. The presence of a deficit in an auditorily similar set probably indicates auditory coding but its absence in a visually similar set may just indicate a lesser degree of confus. ability.

Further interpretive complications may be added by constructing auditorily and visually similar sets using different criteria. While the sets constructed in this paper were developed in order to maximize mean interletter ratings for the whole set, one could choose sets which contain groups of letters highly similar to some set members but not to others (e.g., Vingilis et al., 1977). Such auditorily similar small groupings may function as a coding aid and may explain why Vingilis et al; failed to find the usual performance decrement when an auditorily similar set was compared to a visually similar set. Such findings would not, however, rule out the presence of auditory coding in the situation.

Great care should be taken in interpreting experiments which involve visually and auditorily confusable sets. There are undoubtedly even more complications than have been pointed out here.

\section{REFERENCES}

Connor, J. M., \& Hoyer, R. G. Auditory and visual similarity effects in recognition and recall. Memory \& Cognition, 1976, 4, 261-264.

ConRAd, R. Acoustic confusions in immediate memory. British Journal of Psychology, 1964, 55, 75-84.

GrBson, E. Principles of perceptual learning and development. New York: Appleton-Century-Crofts, 1969.

Vingilis, E., Blake, J., \& Theodor, L. Recognition vs recall of visually vs acoustically confusable letter matrices. Memory \& Cognition, 1977, 5, 146-150.

(Received for publication August 12, 1977; accepted August 19, 1977.) 